Gut, 1975, 16, 665-682

\title{
Studies of intestinal lymphoid tissue
}

\section{Electron microscopic evidence of 'blast transformation' in epithelial lymphocytes of mouse small intestinal mucosa}

\author{
M. N. MARSH'
}

From the Department of Medicine, Southampton University School of Medicine, and the Professorial Medical Unit, Royal South Hants Hospital, Southampton, Hampshire, England

SUMMARY The morphology of epithelial lymphocytes in osmium-fixed, Epon-embedded jejunum of adult mice was studied by light and electron microscopy. Toluidine blue-stained $1 \mu \mathrm{m}$ 'thick' plastic sections were compared with adjacent thin sections, thereby permitting precise ultrastructural identification and description of selected epithelial lymphocytes. Their size and appearances varied considerably, ranging from typical small lymphocytes through medium-sized lymphocytes to large immunoblasts.

A high proportion of medium-sized epithelial lymphocytes (mean diameter $6.9 \pm 1 \cdot 1 \mu \mathrm{m}$ ) contained several lysosomes, extensive Golgi complexes, prominent centrioles and abundant ribosomes. Their appearances, therefore, corresponded directly to mitogen-stimulated lymphocytes. In contrast, immunoblasts were big cells (mean diameter $11.0 \pm 0.8 \mu \mathrm{m}$ ) with large, euchromatic nuclei and prominent nucleoli. The majority had pale-staining, ribosome-studded cytoplasm and thus resembled type $\mathrm{I}$, or $\mathrm{T}$ blasts. Very rarely, densely staining blasts containing ribosomes and well developed rough endoplasmic reticulum were observed; these corresponded to type II or B blasts. These observations indicate that transformation of lymphocytes occurs within the interepithelial cell spaces of the small intestinal mucosa, suggesting that epithelial lymphocytes" are immunocompetent cells which may be responsive to local antigenic stimulation.

For over a century lymphocytes have been observed within the epithelia of many tissues, including the gastrointestinal tract (Schaffer, 1936). It has been suggested that intestinal epithelial lymphocytes either degenerate within the epithelium (Andrew and Sosa, 1947; Shields, Touchon, and Dickson, 1969) or migrate through it, thus to be lost into the intestinal lumen (Wolf-Heidegger, 1939). The functions of epithelial lymphocytes are essentially unknown and only a few studies have been devoted to their fine structure (Andrew, 1965; Meader and Landers, 1967; Toner and Ferguson, 1971).

\footnotetext{
1Address for communications: Department of Medicine, University Hospital of Salford, Eccles Old Road, Salford, Manchester, M6 8HD, UK

Received for publication 17 June 1975.
}

The immunological role of the intestinal mucosa has been increasingly well documented within the last 10 years. It followed that epithelial lymphocytes, rather than entering the epithelium as a prelude to death, might be active participants in the immunological functions of the small intestine. In order to explore this possibility, the light and electron microscopic features of epithelial lymphocytes in adult mouse jejunum were studied and compared with those of other lymphocytes stimulated either in vivo or in vitro (Biberfeld, 1971a; Douglas, 1971). The results of this study suggest that many epithelial lymphocytes are activated cells which undergo 'blast transformation', thus providing some evidence for the view that intestinal epithelial lymphocytes may be responsive to intraluminal antigen. 


\section{Method}

Adult male mice (Charles River Labs, Kent, UK), weighing 25-30 $\mathrm{g}$ and maintained on pellets and tap water, were sacrificed by cervical dislocation. The upper jejunum was then fixed in situ with $1 \% \mathrm{OsO}_{4}$ containing $\mathrm{K}_{2} \mathrm{Cr}_{2} \mathrm{O}_{7}(0.034 \mathrm{~mol}), \mathrm{Nacl}(0.15 \mathrm{~mol})$ and $\mathrm{CaCl}_{2}(0.9 \mathrm{mmol})$. Segments of intestine were carefully excised after one to two min, placed in fresh fixative for a further 60 to $90 \mathrm{~min}$, washed in $10 \%$ buffered formalin ( $\mathrm{pH} 7 \cdot 4)$, dehydrated rapidly in ascending ethanols and embedded in epoxy resin (Epon).

Survey $1 \mu \mathrm{m}$ sections were cut perpendicular to the mucosal surface with glass knives and stained with toluidine blue. Thin sections, adjacent to selected areas of $1 \mu \mathrm{m}$ sections, were cut with a diamond knife on a Reichert OMU-3 ultramicrotome and examined in a Philips EM300 electron microscope. The areas chosen from $1 \mu \mathrm{m}$ 'thick' light microscope sections were thus precisely identified at the ultrastructural level, thereby permitting the fine structure of individually selected epithelial lymphocytes to be detailed.
The diameters of 1000 epithelial lymphocytes, viewed in random $1 \mu \mathrm{m}$ toluidine blue-stained Epon sections with a $\times 100$ oil-immersion objective, were measured with a calibrated ocular graticule.

\section{Results}

Epithelial lymphocytes varied considerably in size and shape and displayed moderately stained nuclei and pale cytoplasm (fig 1). Their pleomorphism was also reflected in the asymmetrical distribution of their diameters (fig 2). The majority of cells ( 90\%) were approximately $5-9 \mu \mathrm{m}$ in diameter (mean \pm SD $6.9 \pm 1 \cdot 1)$ indicating that epithelial lymphocytes are predominantly medium-sized lymphocytes.

Some, however, were greater than $10 \mu \mathrm{m}$ in diameter (mean \pm SD 11.0 \pm 0.8 ) and were therefore considered to be immunoblasts. Although representing only $5 \%$ of the sample of epithelial lymphocytes measured, they were easily recognized in $1 \mu \mathrm{m}$ sections of Epon-embedded jejunum (fig 1).

Among the remaining $5 \%$ epithelial lymphocytes ( $<5.5 \mu \mathrm{m}$ in diameter) were typical small lymphocytes (Bessis, 1961) with round, heterochromatic

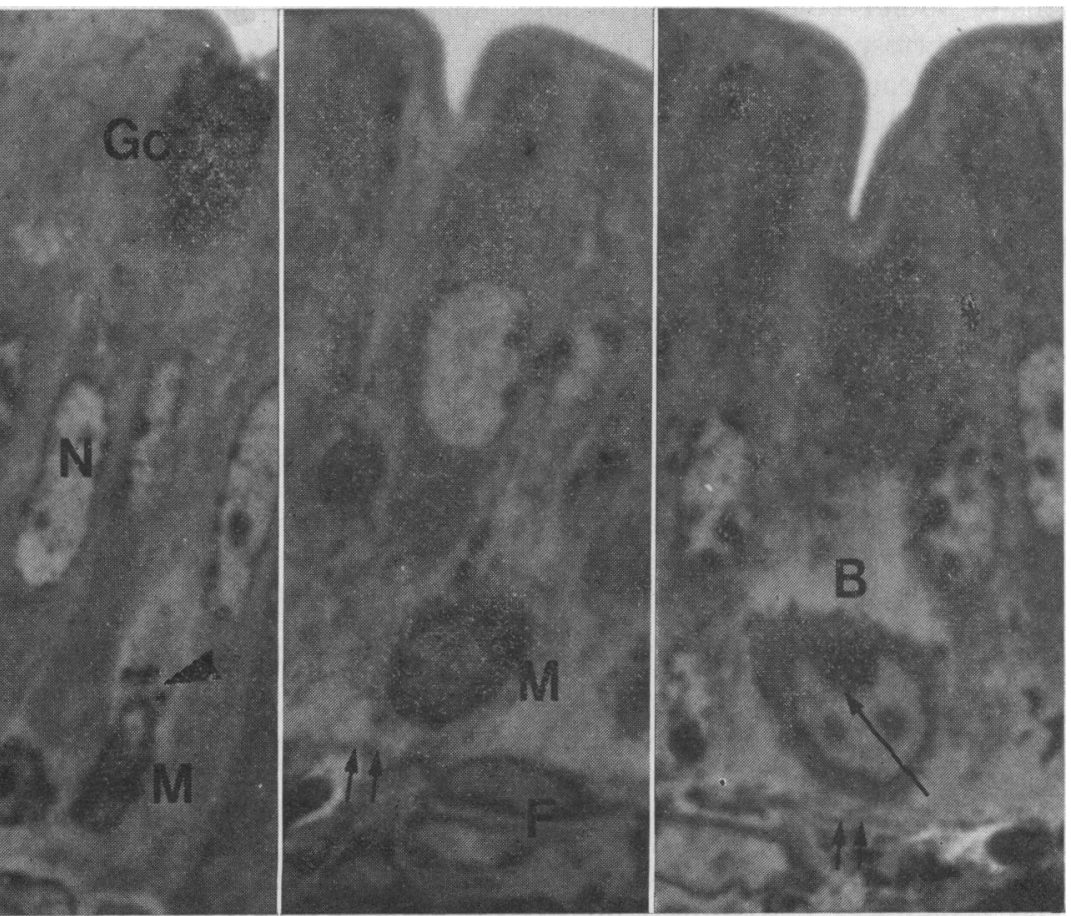

Appearances (left to right) indicate progression of morphological changes during transformation. Nuclei of small lymphocytes are round and compact, with or without a nucleolus. Those of medium-sized epithelial lymphocytes are larger, show more marginated heterochromatin and multiple nucleoli. Immunoblast nuclei are pale, predominantly euchromatic and contain very large nucleoli (long arrow). Cytoplasm of medium-sized epithelial lymphocytes contains numerous densely staining inclusions (arrow head) which are less apparent in blast cytoplasm $(\times 1450)$.

Fig 1 These three sections of Epon-embedded mouse jejunum illustrate the morphological variations of small $(S)$, medium-sized $(M)$, epithelial lymphocytes and an immunoblast $(B)$. They predominantly lie basally within the epithelium, near basement membrane (arrows), and are easily distinguished from columnar epithelial cells (nuclei, $N$ ) and goblet cells (Go). Subepithelial fibroblast $(F)$. 


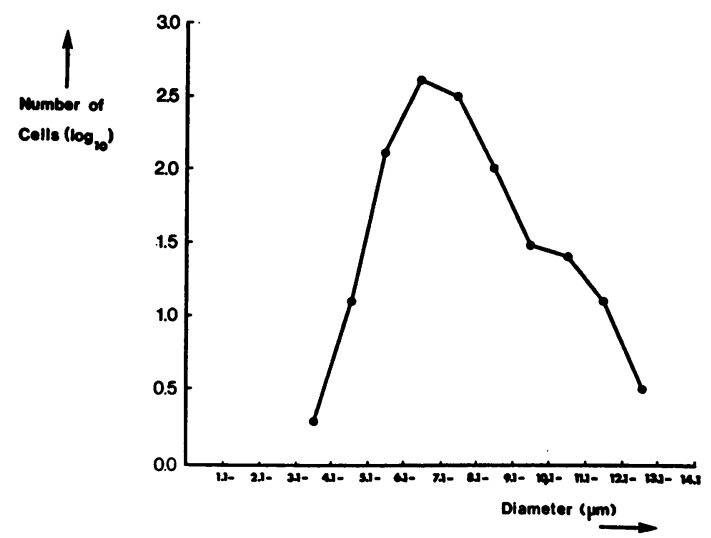

Fig 2 This graph illustrates diameters of 1000 epithelial lymphocytes in villous epithelium of adult mouse jejunum. Diameters (grouped in $1 \mu \mathrm{m}$ increments) are expressed along the abscissa and the number of epithelial lymphocytes within each group (as $\left.\log _{10}\right)$ are plotted along the ordinate. The marked asymmetry of the distribution reflects the morphological heterogeneity of epithelial lymphocytes ( fig 1). nuclei and scanty cytoplasm containing only sparse organelles (figs 1,3 ). Other cells in this group were intermediary between small lymphocytes and the majority of medium-sized epithelial lymphocytes, displaying increased cytoplasmic bulk and nuclei containing prominent nucleoli and an increase in euchromatin (fig 4).

The cytoplasm of medium-sized epithelial lymphocytes showed extensive growth and development of intracellular organelles (figs 1, 5, 6). Such cells were characterized by multiple, deep-staining inclusions (fig 1) varying in size $(0 \cdot 2-0 \cdot 8 \mu \mathrm{m}$ diameter: occasionally $>1 \mu \mathrm{m}$ ) and clustered together adjacent to the nucleus, often within an indentation of the nuclear envelope (figs 6a, 6b). Bounded by a single membrane, they contained either homogeneous, osmiophilic material (figs $6 \mathrm{a}, 6 \mathrm{~b}$ ) or heterogeneous elements (figs 5e, 6a): multivesicular bodies were also present within the cytoplasm (figs $5 \mathrm{c}$, $5 \mathrm{~d}$, 6b). Together these inclusions were identical to the vacuolar lysosomal systems containing acid hydrolase (Daems, Wisse, and Brederoo, 1969; Wattiaux, 1969) described in other cells, including transforming lymphocytes (Parker, Wakasa, and Lukes, 1965; Biberfeld, 1971b).

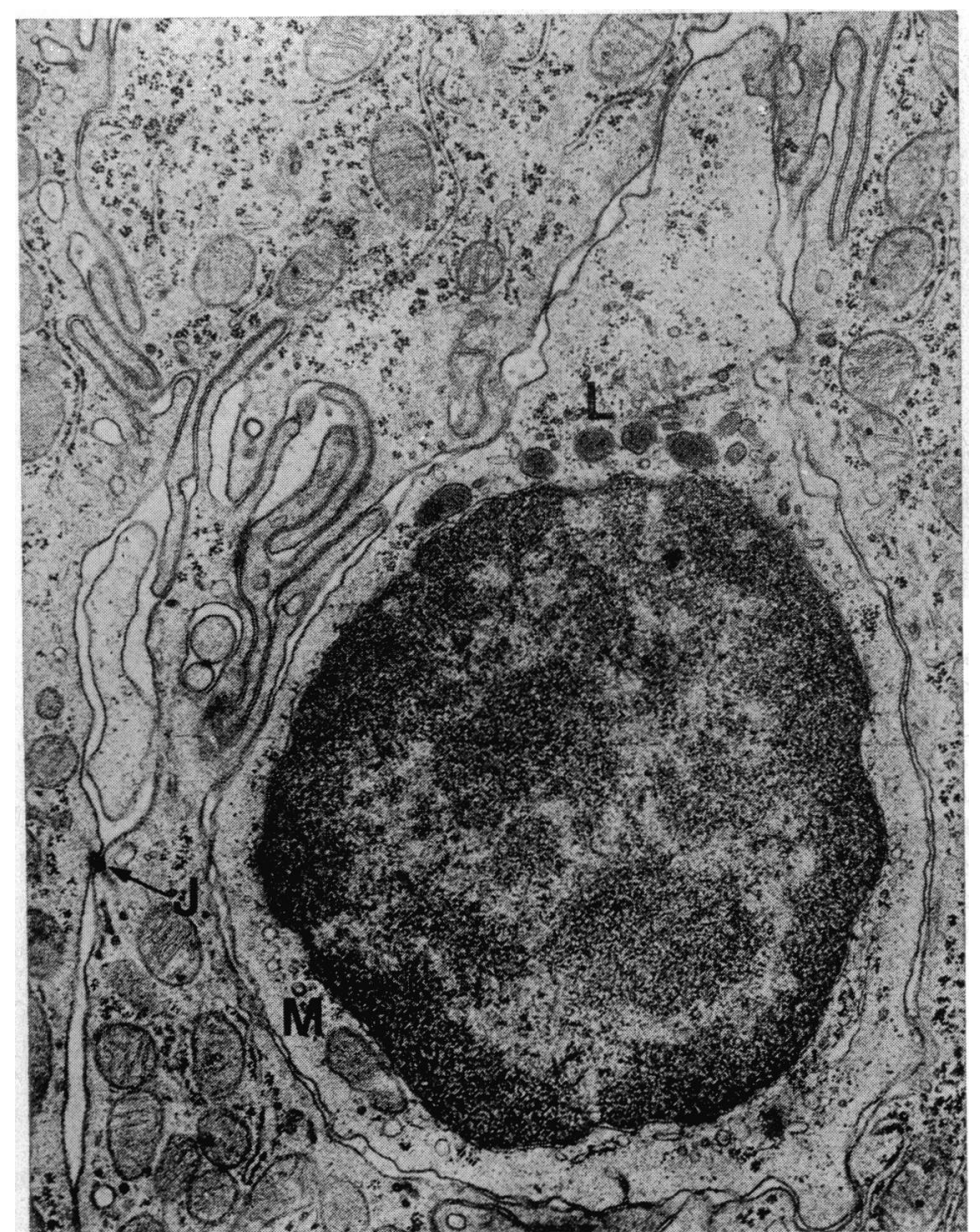

Fig 3 This electron micrograph illustrates the typical fine structure of a small epithelial lymphocyte. The compact, heterochromatic nucleus is enclosed by a rim of cytoplasm containing scattered vesicles, single ribosomes, small lysosomes $(L)$, and occasional mitochondria $(M)$. Its undulating plasma membrane only associates intermittently with lateral epithelial cell membranes without forming specialized junctions, eg, desmosomes between adjacent epithelial cells $(J)(\times 15800)$. 


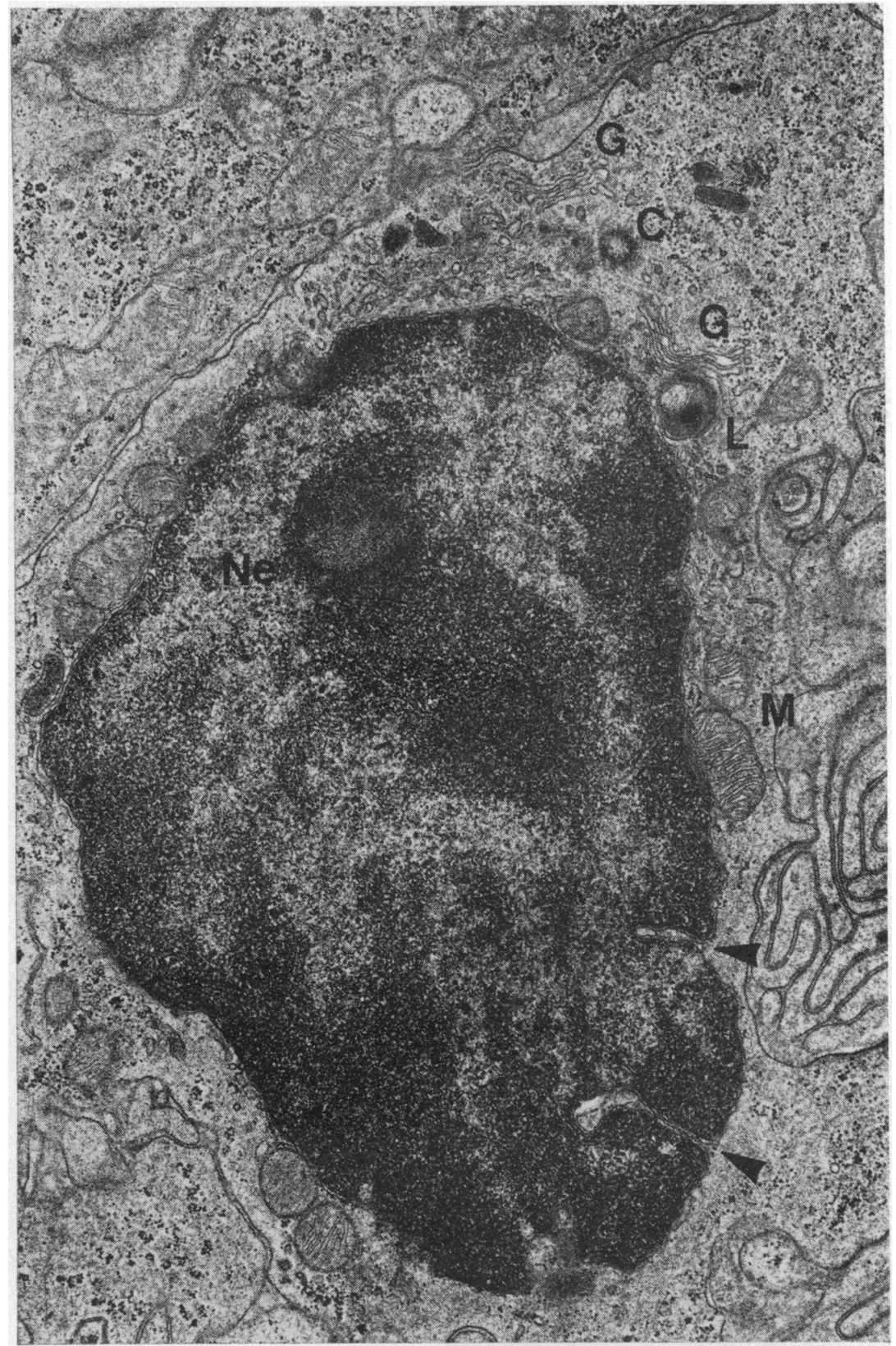

Fig 4 This medium-sized lymphocyte shows certain ultrastructural differences from that in figure 3. Nuclear membrane is irregular and deeply invaginated: there is some increase in euchromatin and a prominent nucleolus (Ne). Cytoplasm is more abundant, containing two well defined Golgi stacks $(G)$, one of which is located adjacent to plasma membrane; a centriole $(C)$, several mitochondria $(M)$, and numerous lysosomes (L). This cell is clearly transitional between small epithelial lymphocytes and the medium-sized cells illustrated in figures 5 and $6(\times 18200)$.

The Golgi complex was well developed in mediumsized epithelial lymphocytes (figs $5 \mathrm{a}, 6 \mathrm{~b}$ ) comprising stacks of at least four parallel lamellae, some of which were dilated either terminally or throughout their length (figs 5a, 6b). Lamellae were frequently located at the nuclear poles (fig $6 \mathrm{~b}$ ) although others were scattered throughout the cytoplasm or adjacent to the plasma membrane (fig 4). Vesicles of varying appearance were usually abundant within the area of the main Golgi complex (fig $6 \mathrm{~b}$ ) some appearing to arise from its smooth tubular elements (figs 5a, 6b). Other vesicles had 'fuzzy' limiting membranes (fig 6b) and thus could have originated from endocytic vesicles formed in minute invaginations of the cell membrane (fig 5b). Prominent within the Golgi region, in favourable sections, were well developed centrioles often orientated perpendicular to each other (figs 5a, 6a). 


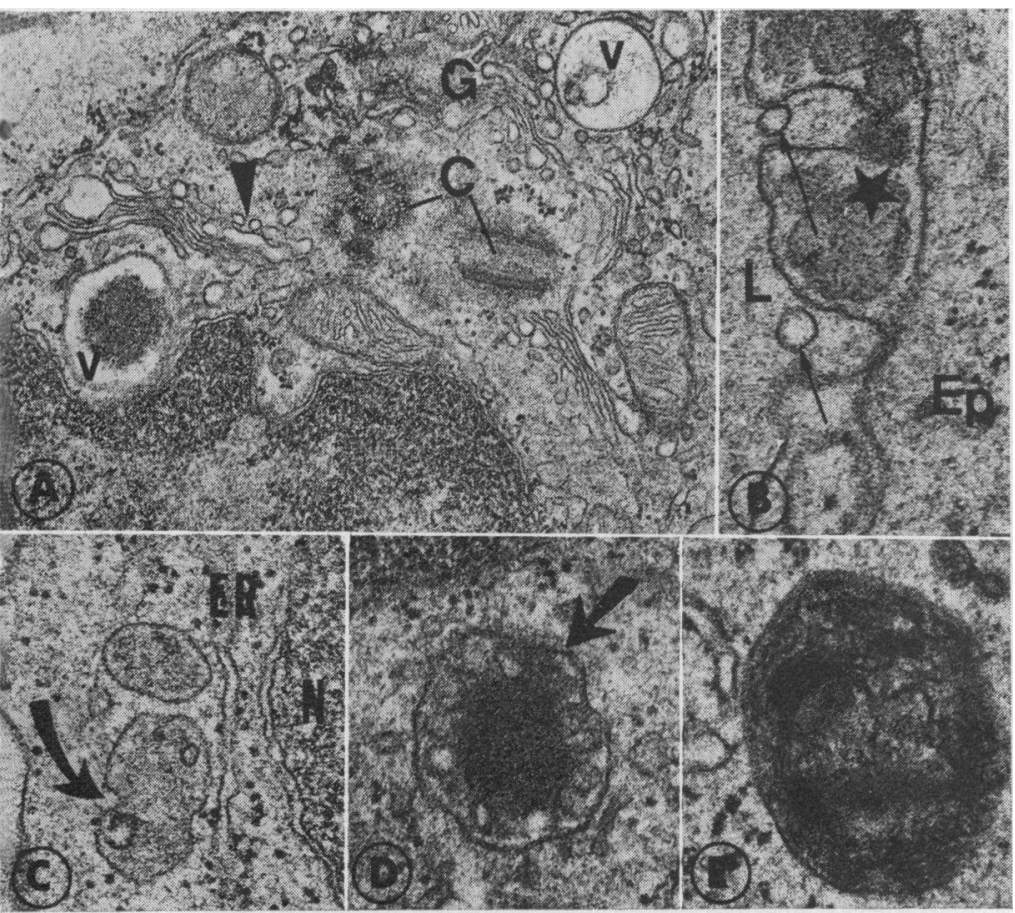

A This perinuclear region contains prominent lamellar and vesicular (arrow head) Golgi elements: heterogeneous vacuoles $(V)$, enclosed by single membranes and well developed centrioles $(C)$, set in dense ground substance. One centriole is cut transversely, thereby demonstrating the typical $9 \times 3$ arrangement of its constituent tubular walls $(\times 25000)$.

B This detail shows the formation of endocytic vesicles (arrows) in minute invaginations of lymphocyte $(L)$ plasmalemma. * denotes flocculent material between lymphocyte and adjacent epithelial cell $(E p)(\times 46100)$.

$\mathrm{C}$ Arrow indicates incorporation of endocytic vesicle into multivesicular body. Profiles of ribosome-studded endoplasmic reticulum ( $r E R)$ lie adjacent to nuclear membrane $(N)(\times 55300)$.

D This micrograph shows another type of multivesicular body (arrow) with a central dense body and satellite vesicles $(\times 40000)$.

$\mathrm{E}$ This is a well developed lysosomal particle containing heterogeneous material (× 36500$)$.

Fig 5 This composite electron micrograph illustrates features of the ultrastructural organization of transforming epithelial lymphocytes.

Mitochondria were larger and more abundant in medium-sized epithelial lymphocytes (figs 4,6 ) than in small lymphocytes (fig 3). Scattered narrow profiles of rough endoplasmic reticulum were present in most cells (figs 5c, 6b) and were usually associated with Golgi membranes (fig 6b), mitochondria (fig 6b) or the outer leaflet of the nuclear membrane (fig 5c). This organelle was, therefore, not extensively developed, while conversely single and clustered ribosomes were more regularly observed throughout the cytoplasm (fig 4) except for the Golgi zone (figs 4, 6a, 6b).

While the nuclei of medium-sized epithelial lymphocytes were either circular or fusiform in section, many were lobulated and showed deep clefts and indentations in their limiting membranes (figs 4, 6). Variable amounts of heterochromatin were located against the nuclear membrane whereas euchromatin, in contrast to small lymphocytes, was increased: nucleoli were prominent and often multiple (fig 1).

In contrast, immunoblasts were characterized by their increased diameter and large, euchromatic nuclei (fig 1): their cytoplasm was most often lightly or moderately stained (figs, 1,7 ) although occasion- ally blasts with densely stained cytoplasm were seen (fig 8). Thus, in $1 \mu \mathrm{m}$ toluidine-blue-stained Epon sections, the appearance of immunoblasts was striking and distinct from other epithelial lymphocytes (fig 1). At the ultrastructural level, the cytoplasm of pale-staining blasts (fig 7) was packed with clusters of ribosomes (fig 7-EM inset): rough endoplasmic reticulum was extremely scanty. In contrast, the cytoplasm of deeply staining blasts contained both polyribosomes and also more extensively developed rough end plasmic reticulum (fig 8-EM insets). Furthermore, small clusters of three or four ribosomes were attached to the outer leaflet of the nuclear membrane which, in places, was in continuity with cytoplasmic rough endoplasmic reticulum (fig 8--EM inset 1). Mitochondria either appeared normal (fig 7) or were markedly enlarged with dilated cristal membranes (fig 8-EM insets 1,2).

The nuclei of these blasts contained only scanty heterochromatin: nucleoli were very large and located adjacent to the internal surface of the nuclear membrane (figs $1,7,8$ ): in addition, nuclear 'pores' were both prominent and more numerous than in smaller epithelial lymphocytes (fig 8-EM inset 1). 
Fig 6 These two electron micrographs illustrate further characteristic features of medium-sized epithelial lymphocytes labelled $(M)$ in figure 1.

A Here, an osmiophilic vesicle indents nuclear membrane: adjacent are other heterogeneous, lysosome-like inclusions bounded by single membranes-a mitochondrion is possibly being engulfed (arrow). 'Chain vesicles' are indicated by curved arrow. Polyribosomes are scattered throughout the cytoplasm $(\times 25200)$.

B This shows an elaborate Golgi system, adjacent to scalloped nuclear membrane. Note large nuclear pore (arrow).

There is a well orientated stack of Golgi lamellae $(G)$, some showing dilatation terminally with formation of smooth-walled vesicles (arrow head): other lamellae are dilated throughout their length. Other 'fuzzy' vesicles ( $F z)$ are abundant within the Golgi zone. Rough endoplasmic reticulum $(E R)$ is associated with mitochondria and Golgi lamellae: there are several mitochondria, some with indistict cristae, and a multivesicular body (MVB). Osmiophilic vesicles $(V)(\times 28000)$. 
Fig 7 Inset: the $1 \mu m$ Epon section (upper left) shows immunoblast $(B)$ with pale-staining cytoplasm located within the jejunal epithelium: arrow head indicates basement membrane. Densely stained lipid $(F)$ lies in adjacent intercellular space and in lamina propria. Epithelial cell nucleus $(N)(\times 1300)$.

The electron micrograph illustrates the fine structure of immunoblast as revealed in a thin section immediately adjacent to $1 \mathrm{\mu m}$ inset. It has a large, euchromatic nucleus and prominent nucleolus (Ne). Its cytoplasm is packed with polyribosomes. Extracellular fat globules $(F)$. Arrow heads indicate basal lamina $(\times 19300)$.

Details within the outlined box $(A)$ are shown at higher magnification (lower left). Arrow indicates prominent nuclear pore: space between double membrane profiles of nuclear envelope are widened. Clustered polyribosomes (Rb) are abundant while rough $(E R)$ is absent $(\times 42800)$. 


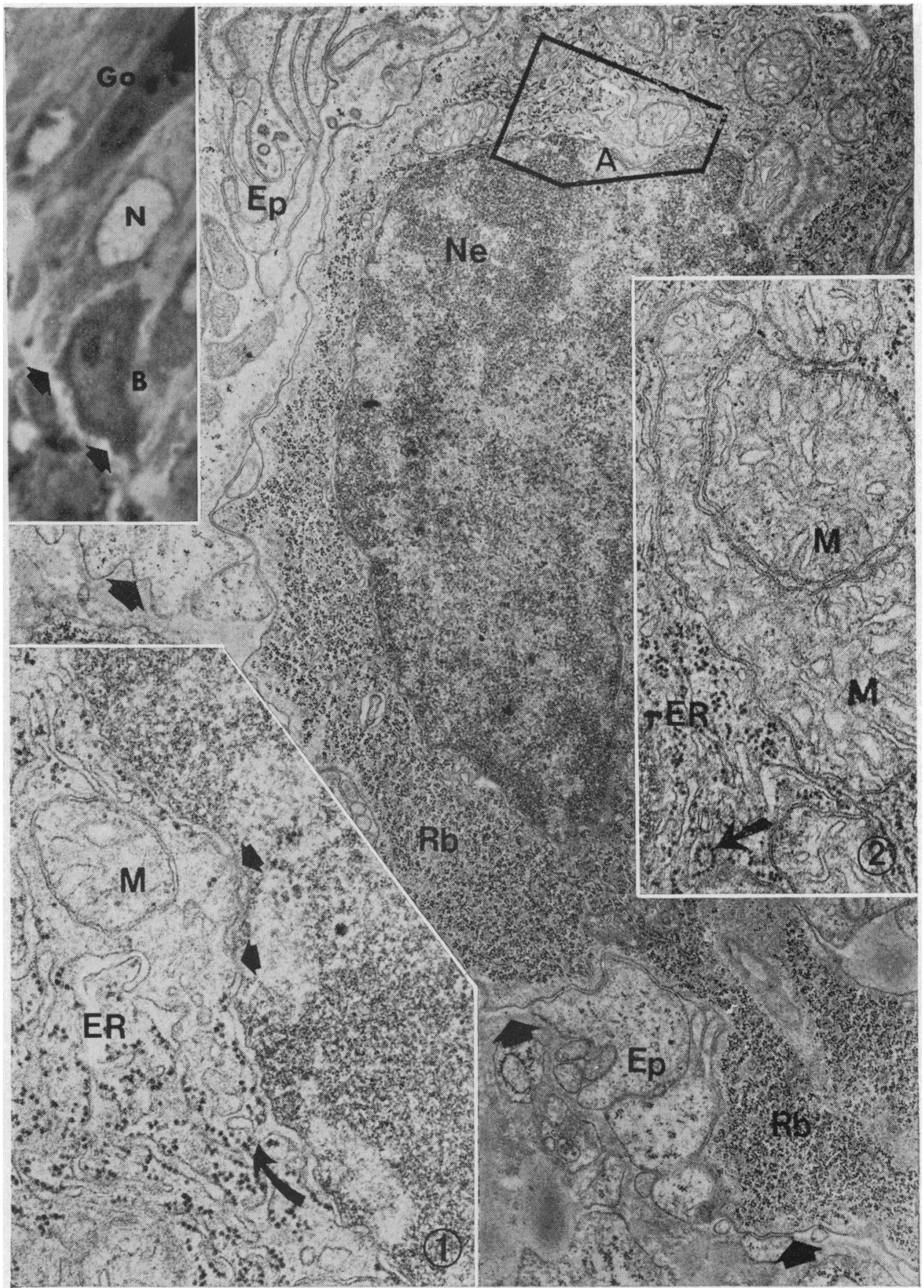

Fig 8 Inset: This 1 um light microscope Epon section (upper left) illustrates a large immunoblast with densely staining cytoplasm $(B)$ within villous epithelium. Note large euchromatic nucleus and prominent nucleolus. Epithelial cell nucleus $(N)$, goblet cell $(G o)$. Basement membrane arrowed $(\times 1300)$.

Electron microscopy of adjacent thin section reveals fine structure of cell $B$ which lies close to epithelial cell cytoplasm (Ep) and basement membrane (arrow heads). Its nucleolus ( $\mathrm{Ne}$ ) abuts against nuclear membrane. Cytoplasm contains abundant ribosomes $(R b)$ but also prominent collections of rough endoplasmic reticulum $(\times 14800)$.

EM inset 1 (lower left) is an enlargement of box $A$ and more clearly shows several dilated ribosome-covered cisternae of endoplasmic reticulum. Note attachment of rough endoplasmic reticulum to nuclear membrane (curved arrow): other linear groups of ribosomes are attached to the exterior nuclear membrane, in the vicinity of two nuclear pores (arrow heads). Mitochondrion $(M)(\times 37800)$.

EM inset 2 (centre right) is part of a deeper section of the same immunoblast, showing well developed rough endoplasmic reticulum and spiral polyribosome (arrow). The two very large mitochondria $(M)$ contain dilated cristae and scattered fine granules $(\times 45000)$. 


\section{Discussion}

These results indicate that in addition to small lymphocytes, the epithelium of adult mouse jejunum contains approximately $90 \%$ medium-sized lymphocytes and a smaller proportion $(5 \%)$ of immunoblasts. These findings agree with those of Rudzik and Bienenstock (1974) who have successfully isolated epithelial lymphocytes from human and rabbit small intestinal mucosa.

Many epithelial lymphocytes displayed the ultrastructural features of 'activated' or transforming lymphocytes. In considering the evidence for this view, it was shown that increases in cytoplasmic bulk were accompanied by the development of numerous lysosome-like bodies, other vacuolar inclusions and extensive elaboration of Golgi lamellae. There were also parallel changes in nuclear morphology ranging from small, densely stained heterochromatic nuclei to large, pale-staining and predominantly euchromatic structures containing multiple, prominent nucleoli (fig 1).

The morphological changes accompanying transformation, both of human (Biberfeld, 1971a; Douglas, 1971 ; Biberfeld, 1971b; Douglas, Hoffman, Borjeson, and Chessin, 1967) and animal (Knight, Ling, Sell, and Oxnard, 1965; Janossy, Shohat, Greaves, and Dourmashkin, 1973) lymphocytes, have been extensively documented, thus providing a firm basis for evaluating the morphological changes described above for intestinal interepithelialspace lymphocytes. Mitogen-induced transformation of lymphocytes in vitro initiates endocytosis, the formation of multivesicular bodies and lysosomes (Parker et al, 1965; Biberfeld, 1971b) and an increase in acid hydrolase activity (Biberfeld, 1971b; Hirschhorn, Brittinger, Hirschhorn, and Weissmann, 1968). Similar changes have been observed in vivo in contact-sensitized lymph node cells (Diengdoh and Turk, 1965). Transformed cells are also characterized by extensive development of the Golgi organelle, the presence of well defined centrioles and the formation of clustered polyribosomes (Biberfeld, 1971a; Douglas, 1971; Janossy et al, 1973; Douglas, 1972). Such changes are known to reflect increased nuclear and cytoplasmic activity (Douglas, 1971; Douglas, 1972) resulting in Ig production, thus indicating that mitogen-induced activation directly simulates the effect of antigen in vivo (Douglas, 1971; Douglas, 1972; Greaves and Janossy, 1972).

Thus, despite the limitations inherent in interpreting selected micrographs, it could be argued that the appearances of many epithelial lymphocytes were indicative of their potential for growth and blast cell formation, presumably in response to local antigenic stimulation from the intestinal lumen. Clearly such interpretations require further detailed study. What is evident from this study is that epithelial lymphocytes do not resemble degenerating lymphocytes (Trowell, 1966; Hummeler, Harris, Harris, and Farber, 1972) so that it is difficult to uphold the view that they are primarily destroyed within the epithelium (Shields et al, 1969; Andrew, 1965).

Other studies of lymphocytes cultured in the presence of selective mitogens have provided morphological criteria for distinguishing between activated B and T lymphocytes (Douglas, 1971; Janossy et al, 1973; Douglas, 1972; Greaves and Janossy, 1972). Most of the activated epithelial lymphocytes observed during this study in jejunal epithelium, including the blasts with pale-staining, ribosome-studded cytoplasm (fig 7), closely resemble the appearance of stimulated $T$ cells. Furthermore, these observations agree with those of Guy-Grand and colleagues (Guy-Grand, Griscelli, and Vassalli, 1974) who concluded that the majority of epithelial lymphocytes are apparently thymus derived, or $\mathbf{T}$ lymphocytes. However, a proportion appear to be B lymphocytes (Ferguson and Parrott, 1972; Parrott and Ferguson, 1974) and the blasts with densely staining cytoplasm containing polyribosomes and moderately developed rough endoplasmic reticulum (fig 8) could conceivably be regarded as type II (Douglas et al, 1967; Janossy et al, 1973) or B blasts. It is also possible that the latter type of blast might ultimately develop into more typical plasma cells, as has been shown for lymphocytes with similar morphology during other experimental conditions (Movat and Fernando, 1965; de Petris and Karlsbad, 1965; Storb, Chambers, Storb, and Weiser, 1967; Sordat, Sordat, Hess, Stoner, and Cottier, 1970; Gudat, Harris, Harris, and Hummeler, 1970).

It remains to be established whether the interepithelial cell spaces enhance contact between epithelial lymphocytes and antigenic material entering the mucosa from the lumen. Whether lymphocytes migrate into the epithelium only in response to such antigen seems unlikely (Ferguson and Parrott, 1972; Parrott and Ferguson, 1974; Guy-Grand et al, 1974) but of all the various cell types present within the lamina propria, it is remarkable that only lymphocytes predominantly gain access to the epithelium. This study shows that once inside the villous epithelium many lymphocytes 'transform' and become blasts. The significance of this transformation and the ultimate pattern of differentiation and migration of epithelial lymphocytes awaits further elucidation.

References

Andrew, W. (1965). Lymphocyte transformation in epithelium. J. nat. Cancer Inst., 35, 113-137. 
Andrew, W., and Sosa, J. M. (1947). Mitotic division and degeneration of lymphocytes within cells of intestinal epithelium in young and in adult white mice. Anat. Rec. 97, 63-97.

Bessis, M. C. (1961). Ultrastructure of lymphoid and plasma cells in relation to globulin and antibody formation. Lab. Invest., 10, 1040-1067.

Biberfeld, P. (1971a). Morphogenesis in blood lymphocytes stimulated with phytohaemagglutinin (PHA). Acta path. microbiol. scand., A. Suppl. 223, 1 .

Biberfeld, P. (1971b). Endocytosis and lysosome formation in blood lymphocytes transformed by phytohemagglutinin. J. ultrastruct. Res., 37, 41-68.

Daems, W. T., Wisse, E., and Brederoo, P. (1969). Electron microscopy of the vacuolar apparatus. In Lysosomes in Biology and Pathology, edited by J. T. Dingle and H. B. Fell, vol. 1, p. 64. North-Holland, Amsterdam.

Diengdoh, J. V., and Turk, J. L. (1965). Immunological significance of lysosomes within lymphocytes in vivo. Nature (Lond.), 207, 1405-1406.

Douglas, S. D., Hoffman, P. F., Borjeson, J., and Chessin, L. N. (1967). Studies on human peripheral blood lymphocytes in vitro: III. Fine structural features of lymphocyte transformation of pokeweed mitogen. J. Immunol., 98, 17-30.

Douglas, S. D. (1971). Human lymphocyte growth in vitro: morphologic, biochemical and immunologic significance. Int. Rev. exp. Path., 10, 41-114.

Douglas, S. D. (1972). Electron microscopic and functional aspects of human lymphocyte response to mitogens. Trans. Rev., 11, 39-59.

Ferguson, A., and Parrott, D. M. V. (1972). The effect of antigen deprivation of thymus-dependent and thymus-independen lymphocytes in the small intestine of the mouse. Clin. exp. Immunol., 12, 477-488.

Greaves, M., and Janossy, G. (1972). Elicitation of selective T and B lymphocyte responses by cell surface binding ligands. Transplant. Rev., 11, 87-130.

Gudat, F. G., Harris, T. N., Harris, S., and Hummeler, K. (1970). Studies on antibody-producing cells. I. Ultrastructure of $19 \mathrm{~S}$ and 7S antibody-producing cells. J. exp. Med., 132, 448-474.

Guy-Grand, D., Griscelli, C., and Vassali, P. (1974). The gut-associated lymphoid system: nature and properties of the large dividing cells. Europ. J. Immunol., 4, 435-443.

Hirschhorn, R., Brittinger, G., Hirschhorn, K., and Weissmann, G. (1968). Studies on lysosomes. XII. Redistribution of acid hydrolases in human lymphocytes stimulated by phytohaemagglutinin. J. Cell Biol., 37, 412-423.

Hummeler, K., Harris, T. N., Harris, S., and Farber, M. B. (1972). Studies on antibody-producing cells. IV. Ultrastructure of plaque-forming cells of rabbit lymph. J. exp. Med., 135, 491-502.
Janossy, G., Shohat, M., Greaves, M. F., and Dourmashkin, R.(1973). Lymphocyte activation. IV. The ultrastructural pattern of the response of mouse $T$ and $B$ cells to mitogenic stimulation in vivo. Immunology, 24, 211-227.

Knight, S., Ling, N. R., Sell, S., and Oxnard, C. E. (1965). The transformation in vitro of peripheral lymphocytes of some laboratory animals. Immunology, 9, 565-574.

Meader, R. D., and Landers, D. F. (1967). Electron and light microscopic observations on relationships between lymphocytes and intestinal epithelium. Amer. J. Anat., 121, 763-774.

Movat, H. Z., and Fernando, N. V. P. (1965). The fine structure of the lymphoid tisssue during antibody formation. Exp. molec. Path., $4,155-188$.

Parker, J. W., Wakasa, H., and Lukes, R. J. (1965). The morphologic and cytochemical demonstration of lysosomes in lymphocytes incubated with phytohemagglutinin by electron microscopy. Lab. Invest., 14, 1736-1743.

Parrott, D. M. V., and Ferguson, A. (1974). Selective migration of lymphocytes within the mouse small intestine. Immunology, 26, 571-588.

de Petris, S., and Karlsbad, G. (1965). Localization of antibodies by electron microscopy in developing antibody-producing cells. J. Cell Biol., 26, 759-778.

Rudzik, O., and Bienenstock, J. (1974). Isolation and characteristics of gut mucosal lymphocytes. Lab. Invest., 30, 260-266.

Schaffer, J. (1936). Leukocyten in Epithel. In Handbuch der mikrpischen Anatomie des Menschen, edited by von Mollendorf, p. 92. Springer, Berlin.

Shields, J. W., Touchon, R. C., and Dickson, D. R. (1969). Quantitative studies on small lymphocyte disposition in epithelial cells. Amer. J. Path., 54, 129-145.

Sordat, B., Sordat, M., Hess, M. W., Stoner, R. D., and Cottier, H. (1970). Specific antibody within lymphoid germinal center cells of mice after primary immunization with horseradish peroxidase. A light and electron microscopic study. J. exp. Med., 131, 77-91.

Storb, U., Chambers, V., Storb, R., and Weiser, R. S. (1967). Antibody-carrying cells in the immune response II. Ultrastructure of 'rosette'-forming cells. J. reticuloendothel. Soc., 4, 69-83.

Toner, P. G., and Ferguson, A. (1971). Intraepithelial cells in the human intestinal mucosa. J. ultrastruct. Res., 34, 329-344.

Trowell, O. A. (1966). Ultrastructural changes in lymphocytes exposed to noxious agents in vitro. Ouart. J. exp. Physiol., 51, 207-220.

Wattiaux, R. (1969). Biochemistry and function of lysosomes. In Handbook of Molecular Cytology, edited by A. Lima-de-Fariau, p. 1159. North-Holland, Amsterdam.

Wolf-Heidegger, G. (1939). Zur Frage der Lymphocytenwanderung durch das Darmepithel. Z. mikr.-anat. Forsch., 45, 90-103.

\section{Aspects of proliferation and migration of epithelial lymphocytes in the small intestine of mice}

SUMMARY Mice were given either intraperitoneal tritiated thymidine $\left({ }^{3} \mathrm{H}-\mathrm{Tdr}\right)$ or colchicine to study proliferation and migration of intestinal epithelial lymphocytes. Both labelled medium and large lymphocytes ('immunoblasts') were observed throughout the epithelium, crossing the basement membrane and within villous lymphatics for at least seven days after ${ }^{3} \mathrm{H}$-Tdr administration. Epithelial lymphocytes are predominantly young cells, actively dividing at the rate of $1 \%$ per hour. They do not migrate along the villi, unlike epithelial cells, but circulate rapidly through the epithelium, returning to the lamina propria at the rate of approximately 3 epithelial lymphocytes/1000 epithelial cell nuclei/hour. The labelling pattern of epithelial lymphocytes and intralymphatic cells with time was very similar suggesting that epithelial lymphocytes therefore may directly enter adjacent lymphatics and hence gain access to thoracic duct lymph. 